\title{
Channel Estimation and Equalization in the Presence of Timing Offset in MB-OFDM Systems
}

\author{
Y. Li and H. Minn (Contact Author) \\ Department of Electrical Engineering, University of Texas at Dallas \\ \{yinghui.li, hlaing.minn\}@utdallas.edu
}

\begin{abstract}
This paper presents low-complexity preamble-based channel estimation and one-tap frequency-domain equalization for zero-padded MB-OFDM based UWB systems. The required circular convolution property of the received signal is obtained by means of an overlap-add method. The proposed low-complexity channel estimator for each band is developed by first averaging the over-lap-added received preamble symbols within the same band and then applying time-domain least-squares method followed by the discrete Fourier transform. By using the probability density functions of the UWB channel path delays, we also derive the statistics of the UWB channel models, and utilizing them we present several optimization criteria for our proposed channel estimation and equalization in the presence of timing offset. The effectiveness of our proposed methods and optimization criteria are confirmed by computer simulation results.
\end{abstract}

\section{INTRODUCTION}

One of the promising ultra-wide band (UWB) technologies is multi-band orthogonal frequency division multiplexing (MB-OFDM) which has been proposed for the IEEE 802.15.3a standard [1] and adopted in the European standard ECMA368 [2]. MB-OFDM systems are sensitive to synchronization errors [3] and channel estimation errors [4]. The low-powerconsumption requirement of the UWB systems demands lowcomplexity transceivers. All the above features motivate us to develop a novel low-complexity channel estimation and equalization method for the MB-OFDM based UWB systems.

For MB-OFDM systems using conventional cyclic prefix (CP) guard interval, existing OFDM channel estimation methods (e.g., [5], [6]) can be directly applied, or appropriately modified as in [7]. However, the MB-OFDM based UWB systems in [1] and [2] use zero-padded (ZP) guard intervals instead of the CP guard interval. Our paper develops the lowcomplexity channel estimation and equalization for the $\mathrm{ZP}$ MB-OFDM based UWB systems using the preamble in [1] and [2], and optimizes the related parameters. In order to obtain the circular convolution property required in the onetap frequency-domain equalization, we apply an overlap-add (OLA) method after the frequency offset compensation. The proposed low-complexity channel estimator is developed by first averaging the over-lap-added received preamble symbols within the same band and then applying a time-domain leastsquares method followed by the discrete Fourier transform (DFT). The data spreading using the time-frequency codes [1] are considered and an efficient equalization method is proposed. More importantly, we present how to optimize the parameter settings of the proposed channel estimation and equalization methods in the presence of timing offset.

The rest of this paper is organized as follows. Section II describes the signal and channel models. Section III introduces the statistics of the UWB channels. The proposed channel estimation and equalization methods are presented in Section IV and the related parameter optimizations of the proposed methods are described in Section V. Simulation results and discussions are provided in Section VI and the paper is concluded in Section VII.

Notations: The superscripts $T$ and $H$ represent the transpose and the Hermitian transpose, respectively. The $k$-th row, $n$-th column element of a matrix $\boldsymbol{X}$ is denoted by $[\boldsymbol{X}]_{k, n} . E[\cdot]$ represents a statistical expectation. The $N \times N$ unitary DFT matrix is denoted by $\boldsymbol{F}=\left[\boldsymbol{f}_{0}, \boldsymbol{f}_{1}, \ldots, \boldsymbol{f}_{N-1}\right]$, while $\boldsymbol{F}_{K}$ is the sub-matrix of $\boldsymbol{F}$ consisting of the first $K$ columns and $\boldsymbol{F}(:, i: k)$ represents $\left[\boldsymbol{f}_{i}, \boldsymbol{f}_{i+1}, \ldots, \boldsymbol{f}_{k}\right]$. The corresponding frequency-domain variable of a time domain variable $x$ is denoted by $\tilde{x} . \Re\{\cdot\}$ represents the real part of $\{\cdot\}$. The $N \times N$ identity matrix is denoted by $\boldsymbol{I}$ and $\boldsymbol{e}_{i}$ represents the $i$-th column of $\boldsymbol{I}$.

\section{Signal AND Channel Models}

In the MB-OFDM-based UWB systems in [1] and [2], the carrier frequency is hopped within a pre-defined set of carrier frequencies $\left\{f_{q}\right\}$ (corresponding to disjoint frequency bands) from symbol to symbol, according to a time-frequency code. The MB-OFDM system from [1] and [2] has 4 different preamble patterns (for 4 pico-nets), each associated with a different time-frequency code. Each preamble pattern is constructed by successively repeating a time-domain preamble sequence (symbol) over 21 periods. The structure of the preamble pattern 1 in each of the three different bands are shown in Fig. 1 (see [1] [8] for other patterns).

In the MB-OFDM-based systems from [1] and [2], ZP guard intervals ( $N_{\text {pre }}$ prefix and $N_{\text {suf }}$ suffix zero samples; $N_{g}=N_{\text {pre }}+N_{\text {suf }} ; M=N+N_{g}$ ) are used instead of the conventional CP guard interval. Between $i$-th and $(i+1)$-th OFDM symbols in [1] and [2], there are $N_{g}=37$ zero samples which are divided as $N_{\text {suf }}=32$ suffice samples and $N_{\text {pre }}=5$ prefix samples. The preamble sample index 0 corresponds to the first non-zero sample of the first preamble symbol. Denote the low-pass-equivalent time-domain samples (sampled at $N / T=1 / T_{s}, N$ times the sub-carrier spacing) transmitted in the $q$-th frequency band by $\left\{s_{q}(k)\right\}$. During the $m$-th symbol 
interval, i.e., $k \in\left[m M-N_{\text {pre }},(m+1) M-N_{\text {pre }}-1\right],\left\{s_{q}(k)\right\}$ can be all zeros if no signal is transmitted in the $q$-th band.

The IEEE 802.15.3a UWB RF channel model described in [9] is given by

$$
h_{\mathrm{RF}}(t)=X \sum_{l=0}^{L_{h}} \sum_{k=0}^{K} \alpha_{k, l} \delta\left(t-T_{l}-\tau_{k, l}\right)
$$

where $T_{l}, \tau_{k, l}$, and $X$ are random variables representing the delay of the $l$-th cluster, the delay (relative to the $l$-th cluster arrival time) of the $k$-th multipath component of the $l$-th cluster, and the log-normal shadowing, respectively. The channel coefficients are defined as a product of small-scale and large-scale fading coefficients, i.e., $\alpha_{k, l}=p_{k, l} \xi_{l} \beta_{k, l}$ where $p_{k, l}$ takes on equiprobable \pm 1 to account for signal inversion due to reflections, and $\left\{\xi_{l} \beta_{k, l}\right\}$ are log-normal distributed path gains. Then we have

$$
\begin{aligned}
E\left[\alpha_{k, l}\left(T_{l}, \tau_{k, l}\right)\right] & =0 \\
E\left[\left|\alpha_{k, l}\left(T_{l}, \tau_{k, l}\right)\right|^{2}\right] & =\Omega_{0} e^{-T_{l} / \Gamma} e^{-\tau_{k, l} / \gamma} .
\end{aligned}
$$

Details of the channel models are referred to [9].

We consider a low-pass-equivalent system that absorbs the carrier-frequency hopping into the CIR. The sample-spaced low-pass-equivalent CIR for the $q$-th band is given by

$$
h_{q}(n)=X \sum_{l=0}^{L_{h}} \sum_{k=0}^{K} \alpha_{k, l} e^{-j 2 \pi f_{q}\left(T_{l}+\tau_{k, l}\right)} p\left(n T_{s}-T_{l}-\tau_{k, l}-t_{0}\right)
$$

where the effect of the combined transmit and receiver filter with the impulse response $p(t)$ whose span is $\left[-t_{0}, t_{0}\right]$ has been included in the CIR and the delay $t_{0}$ is inserted for the causality.

Define $\left\{t_{l}^{q}\right\}=\left\{t_{l}^{q}(i): i=0, \ldots, N^{\prime}-1 ; l=1, \ldots, L_{q}\right\}$ where $\left\{t_{l}^{q}(i): i=0,1, \ldots, N^{\prime}-1\right\}$ denotes the time-domain sample index set corresponding to the $l$-th non-zero preamble symbol period in the $q$-th band with $N \leq N^{\prime} \leq N+N_{\text {suf. }} L_{q}$ is the number of nonzero preamble symbols in the $q$-th band and depends on the preamble pattern and the band index $q$. Let $\left\{x_{q}(k)\right\}$ denote the low-pass-equivalent time-domain channel output signal samples corresponding to the $q$-th band. Then the corresponding low-pass-equivalent time-domain received samples $\left\{\bar{r}_{q}\left(t_{l}^{q}(i)\right)\right\}$ in the $q$-th band, in the absence of carrier frequency offset (CFO) estimation error, can be expressed as

$$
\bar{r}_{q}\left(t_{l}^{q}(i)\right)=e^{j \varphi} x_{q}\left(t_{l}^{q}(i)\right)+n\left(t_{l}^{q}(i)\right)
$$

where $\varphi$ is an arbitrary carrier phase, $\left\{n\left(t_{l}^{q}(i)\right)\right\}$ are zero-mean independent and identically-distributed circularlysymmetric complex Gaussian noise samples with variance $\sigma^{2}=E\left\{\left|n\left(t_{l}^{q}(i)\right)\right|^{2}\right\}$. Since the receiver does not know in advance when the preamble will arrive, we denote the lowpass-equivalent received signal as $\left\{r_{q}(k)\right\}$ which contains noise-only samples followed by the received preamble samples $\left\{\bar{r}_{q}(m)\right\}$. The number of the initial noise-only samples is a random variable depending on the arrival time of the preamble and the receiver's sync detection window timing.

The whole interval of the 21 preamble symbols with the sample index set $\left\{t_{l}^{q}(i)\right\}$ is divided into three disjoint parts part-a, part-b, and part-c, as shown in Fig. 1. According to the synchronization method proposed in [10], the sync detection and coarse timing estimation are performed during part-a, and the fine timing estimation is based on part-b of the preamble, while the frequency offset estimation is based on both part$\mathrm{b}$ and part-c. In this paper, our channel estimation method is based on both part-b and part-c of the received preamble.

\section{Statistics of The UWB Channel Models}

We first present the pdf of the delay $Z_{k, l} \triangleq T_{l}+\tau_{k, l}$ for the $k$-th ray of the $l$-th cluster of the UWB channel, from which the pdfs of $T_{l}$ and $\tau_{k, l}$ are obtained.

Note that $T_{0}=0, \tau_{0, l}=0 . Z_{k, l}$ can be expressed as the sum of inter-arrival times of the first $l$ clusters and the first $k$ rays of the $l$-th cluster. Since inter-arrival times of the UWB channel paths are independent exponential random variables, $Z_{k, l}$ is the sum of $(l+k-2)$ independent exponential random variables. The moment generating function of $Z_{k, l}$ is given by

$$
\Phi_{Z_{k, l}}(s)=\left(\frac{\Lambda}{\Lambda-s}\right)^{l}\left(\frac{\lambda}{\lambda-s}\right)^{k}, \forall k, l \text { except } k=l=0 \text {. }
$$

$\Lambda$ and $\lambda$ are the cluster arrival rate and the ray arrival rate, respectively. The $m$-th moment of $Z_{k, l}$ is obtained as

$$
\begin{aligned}
E\left[z_{k, l}^{m}\right] & =\left.\frac{d^{m} \Phi_{Z_{k, l}}(s)}{d s^{m}}\right|_{s=0} \\
& =\sum_{i=0}^{m}\left(\begin{array}{c}
m \\
i
\end{array}\right) \prod_{n=0}^{i-1}(-l-n)(-\Lambda)^{-i} \prod_{u=0}^{m-i-1}(-k-u)(-\lambda)^{-m+i} .
\end{aligned}
$$

Applying the residue theory, we obtain the pdf of $Z_{k, l}$ as

$$
\begin{aligned}
f_{Z_{k, l}}\left(z_{k, l}\right) & =\int_{-\infty}^{\infty} \Phi_{Z_{k, l}}(s) e^{-s z_{k, l}} d s \\
& = \begin{cases}\left(R_{1}+R_{2}\right) \Lambda^{l} \lambda^{k}(-1)^{(l+k)} & k \neq 0 \text { or } l \neq 0 \\
\delta\left(z_{k, l}\right) & k=0 \quad \& l=0\end{cases}
\end{aligned}
$$

where

$$
\begin{aligned}
& R_{1}=\left\{\begin{array}{cc}
(-1)^{l} e^{-\Lambda z_{k, l}} \sum_{i=0}^{l-1} \frac{\prod_{m=0}^{i-1}(k+m)}{i !(l-i-1) !} & k \neq 0 \& l \neq 0 \\
(\Lambda-\lambda)^{(-k-i)} z_{k, l}^{(l-i-1)} u\left(z_{k, l}\right) & k \neq 0 \& l \neq 0 \\
\frac{(-1)^{l}}{(l-1) !} z_{k, l}^{(l-1)} e^{-\Lambda z_{k, l}} u\left(z_{k, l}\right) & k=0 \\
0 & l=0
\end{array}\right. \\
& R_{2}= \begin{cases}(-1)^{k} e^{-\lambda z_{k, l} \sum_{i=0}^{k-1} \frac{\prod_{m=0}^{i-1}(l+m)}{i !(k-i-1) !}} & \\
(\Lambda-\lambda)^{(-l-i)} z_{k, l}^{(k-i-1)} u\left(z_{k, l}\right) & k \neq 0 \& l \neq 0 \\
\frac{(-1)^{k}}{(l-1) !} z_{k, l}^{(k-1)} e^{-\lambda z_{k, l}} u\left(z_{k, l}\right) & k \neq 0 \& l=0 \\
0 & k=0,\end{cases}
\end{aligned}
$$

where $u(\cdot)$ is the unit step function.

Since $\left\{T_{l}\right\}$ and $\left\{\tau_{k, l}\right\}$ are independent, we have

$$
f_{T_{l}, \tau_{k, l}}(x, y)=f_{t_{l}}(x) f_{\tau_{k, l}}(y)
$$

where the pdfs of $T_{l}$ and $\tau_{k, l}$ are related to the pdf of $Z_{k, l}$ as

$$
\begin{aligned}
f_{T_{l}}(x) & =f_{Z_{0, l}}(x) \\
f_{\tau_{k, l}}(y) & =f_{Z_{k, 0}}(y) .
\end{aligned}
$$

Next, we find the covariance matrix of the CIR for each band. Let $L$ denote the maximum length of the sample-spaced CIR vector of each band and $\boldsymbol{h}_{q}$ denote the $L \times 1$ CIR vector of the $q$-th band. Depending on the actual length of each channel realization, $\boldsymbol{h}_{q}$ may contain some zero samples. The covariance matrices of $\left\{\boldsymbol{h}_{q}\right\}$ are the same for all bands as will be apparent in the following. Hence, we simply denote the 


$$
\begin{aligned}
& C_{h}(m, n)=\int_{0}^{\infty} \int_{0}^{\infty} \sum_{l=0}^{L_{h}} \sum_{k=0}^{K} E\left[\left|\alpha_{k, l}\left(T_{l}, \tau_{k, l}\right)\right|^{2}\right] p\left(m T_{s}-T_{l}-\tau_{k, l}-t_{0}\right) p\left(n T_{s}-T_{l}-\tau_{k, l}-t_{0}\right) f_{T_{l}, \tau_{k, l}}\left(T_{l}, \tau_{k, l}\right) d T_{l} d \tau_{k, l} \\
& C_{h}(m, n)=\sum_{l=0}^{L_{h}} \sum_{k=0}^{K} \int_{0}^{T} \int_{0}^{T} \Omega_{0} e^{-T_{l} / \Gamma} e^{-\tau_{k, l} / \gamma} p\left(m T_{s}-T_{l}-\tau_{k, l}-t_{0}\right) p\left(n T_{s}-T_{l}-\tau_{k, l}-t_{0}\right) f_{Z_{0, l}}\left(T_{l}\right) f_{Z_{k, 0}}\left(\tau_{k, l}\right) d T_{l} d \tau_{k, l}
\end{aligned}
$$

covariance matrix of $\boldsymbol{h}_{q}$ by $\boldsymbol{C}_{h}$. From (4), we can calculate the element of $C_{h}$ as ${ }^{1}(14)$, where we have used the statistical independence among $\left\{\alpha_{k, l}\left(T_{l}, \tau_{k, l}\right)\right\}$.

Substituting (8)-(13) and (3) into (14) gives (15), which can be numerically evaluated. We will use the above channel covariance matrix in optimizing our proposed methods. We emphasize that the pdfs of the UWB channel path delays that we present in this section are also useful in calculating or optimizing the energy capture of other UWB systems.

\section{Proposed Channel ESTIMATION AND EQUALIZATION}

The use of zero-amplitude guard samples in MB-OFDM UWB systems instead of conventional CP guard samples saves transmit power, avoids the spectrum ripples and allows a sufficient amount of time for the transmitter and receiver to switch between carrier frequencies. However, the circular convolution property exhibited in CP-based systems is no longer maintained in ZP systems. The frequency-domain onetap equalization, which is much desirable due to its lowcomplexity advantage, requires the circular convolution property. Hence, we first apply an OLA method to the frequencyoffset-compensated received signal to obtain the circular convolution property. Assuming $\Delta_{k}$ is the timing offset, for every $l$-th non-zero OFDM symbol in the $q$-th band, the OLA method adds $M_{0}\left(0 \leq M_{0} \leq N_{\text {suf }}\right)$ samples $\left\{r_{q}\left(\Delta_{k}+l M+\right.\right.$ $\left.N), r_{q}\left(\Delta_{k}+l M+N+1\right), \ldots, r_{q}\left(\Delta_{k}+l M+M_{0}+N-1\right)\right\}$ to $\left\{r_{q}\left(\Delta_{k}+l M\right), r_{q}\left(\Delta_{k}+l M+1\right), \ldots, r_{q}\left(\Delta_{k}+l M+M_{0}-1\right)\right\}$, resulting in an $N \times 1$ vector $\boldsymbol{y}_{q}^{(l)}$ with the $k$-th element given by

$$
y_{q}^{(l)}(k)= \begin{cases}r_{q}\left(\Delta_{k}+l M+k\right)+ & r_{q}\left(\Delta_{k}+l M+k+N\right), \\ & \text { if } 0 \leq k \leq M_{0}-1 \\ r_{q}\left(\Delta_{k}+l M+k\right), & \text { else. }\end{cases}
$$

Note that the OLA length $M_{0}$ need not be the same for the preamble symbol and the data symbol. Hence, we use $M_{p}$ and $M_{d}$ to denote the OLA length for the preamble symbol and the data symbol, respectively.

After applying the OLA method, the preamble symbols in each band are averaged as

$$
\overline{\boldsymbol{y}}_{q}=\frac{1}{L_{q, \mathrm{bc}}} \sum_{l=1}^{L_{q, \mathrm{bc}}} \boldsymbol{y}_{q}^{(l)}
$$

where $L_{q, \mathrm{bc}}$ is the number of non-zero preamble symbols of part-b and part-c in the $q$-th band. After the OLA method, we can assume in developing the CIR estimator that $\overline{\boldsymbol{y}}_{q}$ is approximately the same as the received preamble symbol with circular convolution property under perfect frequency synchronization but with a possible timing offset. Although the maximum number of CIR taps is $L$, we estimate length- $L^{\prime}$

\footnotetext{
${ }^{1}$ We neglect the shadowing parameter $X$ in this paper and hence realizations of $\left\{\alpha_{k, l}\right\}$ are not normalized.
}

CIR to account for a random timing offset and to minimize the channel estimation mean-square error (MSE). The optimization of $L^{\prime}$ will be discussed in Section V-A. We apply the time-domain least-squares channel estimator and obtain the length $L^{\prime}$ CIR vector estimate for the $q$-th band as

$$
\hat{\boldsymbol{h}}_{q}=\left(\boldsymbol{S}^{H} \boldsymbol{S}\right)^{-1} \boldsymbol{S}^{H} \overline{\boldsymbol{y}}_{q}
$$

where $[\boldsymbol{S}]_{k, n}=s((k-n) \bmod N), 0 \leq k \leq N-1,0 \leq n \leq$ $L^{\prime}-1$, and $\{s(m): m=0,1, \ldots, N-1\}^{2}$ are the low-passequivalent time-domain OFDM preamble samples. Further improvements on the channel estimation and timing estimation (e.g., see [11] [12]) are possible at the cost of much larger complexity, but we will not pursue them here since UWB systems demand low-complexity algorithms. The frequencydomain channel estimates are then simply obtained by taking the DFT of $\hat{\boldsymbol{h}}_{q}$ as $\left[\tilde{\hat{h}}_{q}(0), \ldots, \hat{\hat{h}}_{q}(N-1)\right]^{T}=\sqrt{N} \boldsymbol{F}_{L^{\prime}} \hat{\boldsymbol{h}}_{q}$.

In general, data can be transmitted with or without spreading using a time-frequency code. Suppose the $m$-th OFDM data symbol (after spreading if applied) is transmitted in the $q$ th band, and let $\tilde{y}_{q, m}(i)$ denote the corresponding received symbol on the $i$-th subcarrier after applying the OLA method and DFT. If the data are not spread by a time-frequency code, the frequency-domain one-tap equalizer output decision variable for the $i$-th subcarrier data of the $m$-th OFDM data symbol is given by

$$
\tilde{Y}^{(m)}(i)=\tilde{\hat{h}}_{q}(i)^{*} \tilde{y}_{q, m}(i) /\left|\tilde{\hat{h}}_{q}(i)\right|^{2}, \quad i=0,1, \ldots, N-1 .
$$

If data spreading is performed using a time-frequency code [1] [2] such as each data symbol is transmitted on two consecutive symbol intervals using a frequency hopping pattern of $\left[l_{0} n_{0}\right.$ $\left.\begin{array}{llll}l_{1} & n_{1} & l_{2} & n_{2}\end{array}\right]$ (e.g., [ [ $\left.\begin{array}{llllll}1 & 2 & 3 & 1 & 2 & 3\end{array}\right]$ ), then the equalizer output decision variable for the $m$-th data (before spreading) on the $i$-th subcarrier is obtained as

$$
\begin{array}{r}
\tilde{Y}^{(m)}(i)=\frac{\left(\tilde{\hat{h}}_{l_{\xi}}(i)^{*} \tilde{y}_{l_{\xi}, 2 m}(i)+\tilde{\hat{h}}_{n_{\xi}}(i)^{*} \tilde{y}_{n_{\xi}, 2 m+1}(i)\right)}{\left(\left|\hat{\hat{h}}_{l_{\xi}}(i)\right|^{2}+\left|\tilde{\hat{h}}_{n_{\xi}}(i)\right|^{2}\right)} \\
i=0,1, \ldots, N-1
\end{array}
$$

where $\xi=m \bmod 3$ in the band indexes $l_{\xi}$ and $n_{\xi}$.

\section{Optimizing the Proposed Methods}

\section{A. CIR Estimation Window Length and Preamble OLA Length}

In Section III, we define the maximum sample-spaced CIR length as $L$, which depends on the practical channel environment. The channel taps with largest delays may contain negligible channel energy and neglecting them in the CIR estimation will suppress noise from those neglected taps and hence may improve the CIR estimation performance. Hence, in this section, we derive channel estimation MSE obtained with the CIR estimation window length $L^{\prime}$, and find the optimum

\footnotetext{
${ }^{2}$ The band index $q$ is dropped since the preamble symbols are the same.
} 


$$
\begin{aligned}
\tilde{\boldsymbol{x}}_{q}^{\mathrm{ICI}}= \begin{cases}\boldsymbol{F}\left(:,\left(M_{d}: L-\Delta_{k}-1\right)\right) \boldsymbol{H}_{1}\left(M_{d}+\Delta_{k}\right)\left(\boldsymbol{F}\left(:,\left(N-L+M_{d}+\Delta_{k}: N-1\right)\right)\right)^{H} \tilde{\boldsymbol{s}}_{q} & \text { if } \Delta_{k}>0 \& M_{d}+\Delta_{k}<L \\
+\boldsymbol{F}\left(:,\left(N-\Delta_{k}: N-1\right)\right) \boldsymbol{H}_{2}\left(\Delta_{k}\right)\left(\boldsymbol{F}\left(:,\left(0: \Delta_{k}-1\right)\right)\right)^{H} \tilde{\boldsymbol{s}}_{q} & \text { if } \Delta_{k}>0 \& M_{d}+\Delta_{k} \geq L \\
\boldsymbol{F}\left(:,\left(N-\Delta_{k}: N-1\right)\right) \boldsymbol{H}_{2}\left(\Delta_{k}\right)\left(\boldsymbol{F}\left(:,\left(0: \Delta_{k}-1\right)\right)\right)^{H} \tilde{\boldsymbol{s}}_{q} & \\
\boldsymbol{F}\left(:,\left(M_{d}: L-\Delta_{k}-1\right)\right) \boldsymbol{H}_{1}\left(M_{d}+\Delta_{k}\right)\left(\boldsymbol{F}\left(:,\left(N-L+M_{d}+\Delta_{k}: N-1\right)\right)\right)^{H} \tilde{\boldsymbol{s}}_{q} & \text { if } \Delta_{k} \leq 0 \& M_{d} \geq-\Delta_{k} \\
\boldsymbol{F}(:,(0: L-1)) \boldsymbol{H}_{1}(0)(\boldsymbol{F}(:,(N-L: N-1)))^{H} \tilde{\boldsymbol{s}}_{q} & \text { if } \Delta_{k} \leq 0 \& M_{d}<-\Delta_{k} \\
\quad+\boldsymbol{F}\left(:,\left(M_{d}:-\Delta_{k}-1\right)\right) \boldsymbol{x}_{q}\left(N+\Delta_{k}+M_{d}: N-1\right) & \text { if } \Delta_{k}>0 \& M_{d}+\Delta_{k} \geq L\end{cases} \\
E\left[\left|\tilde{\boldsymbol{x}}_{q}^{\mathrm{ICI}}\right|^{2}\right]= \begin{cases}\left.\operatorname{trace}\left(E\left[\boldsymbol{H}_{1}\left(M_{d}+\Delta_{k}\right)\left(\boldsymbol{H}_{1}\left(M_{d}+\Delta_{k}\right)\right)^{H}\right]+E\left[\boldsymbol{H}_{2}\left(\Delta_{k}\right)\left(\boldsymbol{H}_{2}\left(\Delta_{k}\right)\right)^{H}\right]\right)\right) & \text { if } \Delta_{k}>0 \& M_{d}+\Delta_{k}<L \\
\operatorname{trace}\left(E\left[\boldsymbol{H}_{2}\left(\Delta_{k}\right)\left(\boldsymbol{H}_{2}\left(\Delta_{k}\right)\right)^{H}\right]\right) & \text { if } \Delta_{k} \leq 0 \& M_{d} \geq-\Delta_{k} \\
\operatorname{trace}\left(E\left[\boldsymbol{H}_{1}\left(M_{d}+\Delta_{k}\right)\left(\boldsymbol{H}_{1}\left(M_{d}+\Delta_{k}\right)\right)^{H}\right]\right) & \text { if } \Delta_{k} \leq 0 \& M_{d}<-\Delta_{k} \\
\operatorname{trace}\left(E\left[\boldsymbol{H}_{1}(0)\left(\boldsymbol{H}_{1}(0)\right)^{H}\right]\right)-\sum_{i=N+\Delta_{k}+M_{d}}^{N-1} E\left[\left|x_{q}(i)\right|^{2}\right] & \end{cases}
\end{aligned}
$$

$L^{\prime}$ which yields the minimum channel estimation MSE. We assume that fine timing and perfect frequency synchronization have been performed. Hence, we develop our optimization for CIR estimation under perfect synchronization first. Then we incorporate the effect of timing offset into our design.

Recall that we apply the OLA method to obtain the circular convolution property. After applying the OLA method with a window length $M_{p}$ on the received preamble symbol, the MSE of the length- $L^{\prime}$ CIR estimation under perfect synchronization can be derived as

$$
\begin{aligned}
& \operatorname{MSE}_{h}\left(L^{\prime}, M_{p}\right)=\operatorname{trace}\left(\boldsymbol{C}_{h}\right)+\sigma^{2} \operatorname{trace}\left(\boldsymbol{\Upsilon}^{H} \boldsymbol{\Upsilon}(\boldsymbol{I}+\boldsymbol{T})\right) \\
& +\operatorname{trace}\left(\boldsymbol{\Upsilon}^{H} \mathbf{\Upsilon} \overline{\boldsymbol{S}} \boldsymbol{C}_{h} \overline{\boldsymbol{S}}\right)-2 \Re\left\{\operatorname{trace}\left(\boldsymbol{\Upsilon} \overline{\boldsymbol{S}} \boldsymbol{C}_{\mathrm{h}}\right)\right\} . \\
& \text { where } \quad \boldsymbol{T}=\left[\boldsymbol{e}_{1}, \ldots, \boldsymbol{e}_{M_{p}}, \mathbf{0}_{N \times\left(N-M_{p}\right)}\right] \\
& \boldsymbol{\Upsilon}=\left(\boldsymbol{S}^{H} \boldsymbol{S}\right)^{-1} \boldsymbol{S}^{H} \\
& \overline{\boldsymbol{S}}=\boldsymbol{S}_{L}-\overline{\boldsymbol{S}}_{\Delta} \\
& \overline{\boldsymbol{S}}_{\Delta}= \begin{cases}{\left[\begin{array}{cc}
\mathbf{0}_{M_{p} \times M_{p}} & \mathbf{0}_{M_{p} \times\left(L-M_{p}\right)} \\
\mathbf{0}_{\left(L-M_{p}\right) \times M_{p}} & \boldsymbol{S}_{\Delta} \\
\mathbf{0}_{(N-L) \times M_{p}} & \mathbf{0}_{(N-L) \times\left(L-M_{p}\right)}
\end{array}\right],} & \text { if } L>M_{p} \\
\mathbf{0}_{N \times L}, & \text { else }\end{cases} \\
& \boldsymbol{S}_{\Delta}=\left[\begin{array}{cccc}
s(N-1) & s(N-2) & \ldots, & s\left(N-L+M_{p}\right) \\
0, & s(N-1), & \ldots, & s\left(N-L+M_{p}+1\right) \\
\vdots & 0, & \ddots, & \vdots \\
0, & 0, & \ldots, & s(N-1)
\end{array}\right] \text {. }
\end{aligned}
$$

$\left[\boldsymbol{S}_{L}\right]_{k, n}=s((k-n) \bmod N), 0 \leq k \leq N-1,0 \leq n \leq L-1$, is an $N \times L$ matrix. We can obtain the optimum $\left(L^{\prime}, M_{p}\right)$ for the CIR estimation under perfect synchronization as

$$
\left(\left[L^{\prime}\right]_{\text {perfect }},\left[M_{p}\right]_{\text {perfect }}\right)=\underset{\left\{L^{\prime}, M_{p}\right\}}{\arg \min }\left\{\operatorname{MSE}_{h}\left(L^{\prime}, M_{p}\right)\right\}
$$

which can be numerically evaluated where the required $\boldsymbol{C}_{h}(m, n)$ is obtained from (15). In the presence of timing errors, the above window lengths in (27) should be increased accordingly so that the CIR estimation window contains the first $\left[L^{\prime}\right]_{\text {perfect }}$ actual channel taps most of the time. We will discuss this issue in Section VI.

\section{B. Optimizing the Data OLA Length}

The OLA method used to obtain the circular convolution property in ZP systems increases noise power if compared to the CP-based systems since the noise terms are also added up. In this sense, we prefer a smaller value of the OLA length $M_{d}$ (for data) which will decrease the extra noise power. However, the shortening of the OLA length $M_{d}$ may destroy the circular convolution property and in this circumstance the OLA method with one-tap frequency-domain equalizer will introduce the inter-carrier interferences (ICI). For example, with perfect timing, if $M_{d}$ is less than the order of the CIR, there will be ICI. Note that the timing offset also affects the optimal value of $M_{d}$. In this section, we will find the optimum $M_{d}$ which minimizes the combined interference and extra noise power introduced by the OLA method.

After applying the OLA method, the received data signal on the $i$-th subcarrier can be expressed as

$$
\tilde{y}_{q}(i)=\tilde{x}_{q}(i)-\tilde{x}_{q}^{\mathrm{ICI}}(i)+\tilde{n}_{q}^{e}(i)+\tilde{n}(i)
$$

where $\tilde{x}_{q}(i)=\tilde{h}_{q}(i) \tilde{s}_{q}(i)$ with $\tilde{s}_{q}(i)$ being the transmitted symbol on the $i$-th sub-carrier in the $q$-th band, $\tilde{n}(i)$ is the frequency-domain noise sample (the same statistics as in $\mathrm{CP}$ based systems), $\tilde{n}_{q}^{e}(i)$ is the extra frequency-domain noise sample due to the OLA method, and $\tilde{x}_{q}^{\mathrm{ICI}}(i)$ is the ICI term on the $i$-th subcarrier due to the OLA method. Denote the corresponding vectors of $\tilde{s}_{q}(i), \tilde{x}_{q}^{\mathrm{ICI}}(i), \tilde{n}(i)$, and $\tilde{n}_{q}^{e}(i)$ by $\tilde{\boldsymbol{s}}_{q}, \tilde{\boldsymbol{x}}_{q}^{\mathrm{ICI}}, \tilde{\boldsymbol{n}}$, and $\tilde{\boldsymbol{n}}_{q}^{e}$, respectively. Note that we have

$\tilde{\boldsymbol{n}}_{q}^{e}=\boldsymbol{F}\left(:,\left(0: M_{d}-1\right)\right) \boldsymbol{n}\left(\Delta_{k}+l M+N: \Delta_{k}+l M+N+M_{d}-1\right)$.

Then, the ICI vector $\tilde{\boldsymbol{x}}_{q}^{\mathrm{ICI}}$ can be expressed as (30), where

$$
\begin{aligned}
& \boldsymbol{H}_{1}(x)=\left[\begin{array}{cccc}
h_{q}(L-1) & h_{q}(L-2) & \ldots & h_{q}(x) \\
0 & h_{q}(L-1) & \ldots & h_{q}(x+1) \\
\vdots & \vdots & \ddots & \vdots \\
0 & 0 & \ldots & h_{q}(L-1)
\end{array}\right] \\
& \boldsymbol{H}_{2}(x)=\left[\begin{array}{cccc}
h_{q}(0) & 0 & \ldots & 0 \\
h_{q}(1) & h_{q}(0) & \ldots & 0 \\
\vdots & \vdots & \ddots & \vdots \\
h_{q}(x-1) & h_{q}(x) & \ldots & h_{q}(0)
\end{array}\right]
\end{aligned}
$$

Since we choose the same OLA length $M_{d}$ for all bands and the channel covariance matrices are also the same for all bands, the interference plus extra noise power caused by the OLA method in each band can be expressed as

$$
\begin{gathered}
\Xi=E\left[\left|\tilde{\boldsymbol{x}}_{q}^{\mathrm{ICI}}\right|^{2}\right]+E\left[\left|\tilde{\boldsymbol{n}}_{q}^{e}\right|^{2}\right] \\
\text { where } \quad E\left[\left|\tilde{\boldsymbol{n}}_{q}^{e}\right|^{2}\right]=M_{d} \sigma^{2} .
\end{gathered}
$$

Considering independent and identically-distributed data symbols on the subcarriers, we have $E\left[\tilde{\boldsymbol{s}}_{q} \tilde{\boldsymbol{s}}_{q}^{H}\right]=\sigma_{s}^{2} \boldsymbol{I}$. Then, $E\left[\left|\tilde{\boldsymbol{x}}_{q}^{\mathrm{ICI}}\right|^{2}\right]$ is given by (35). Since the channel covariance matrix $C_{h}$ can be obtained from Section III, the elements of $E\left[\boldsymbol{H}_{1}(x)\left(\boldsymbol{H}_{1}(x)\right)^{H}\right]$ and $E\left[\boldsymbol{H}_{2}(x)\left(\boldsymbol{H}_{2}(x)\right)^{H}\right]$ can be obtained as

$$
\begin{aligned}
& \left.E\left[\boldsymbol{H}_{1}(x)\left(\boldsymbol{H}_{1}(x)\right)^{H}\right]\right|_{(m, n)}=\sum_{i=0}^{L-x-\max (m, n)} \boldsymbol{C}_{h}(x+m+i, x+n+i) \\
& \left.E\left[\boldsymbol{H}_{2}(x)\left(\boldsymbol{H}_{2}(x)\right)^{H}\right]\right|_{(m, n)}=\sum_{i=0}^{\min (m, n)-1} \boldsymbol{C}_{h}(m-i, n-i) .
\end{aligned}
$$


Substituting (36), (37) and (35) into (33), we finally obtain the interference plus extra noise power $\Xi$ caused by the OLA method which varies with $M_{d}$ for a fixed $\Delta_{k}$. We obtain the optimal $M_{d}$ that minimizes $\Xi$ as

$$
M_{d}^{*}=\underset{M_{d}}{\arg \min }\left\{\Xi\left(M_{d}\right)\right\}
$$

which can be numerically solved easily.

\section{Simulation Results And Discussions}

We use the simulation parameters as specified in [1] and [2]: $N=128, N_{\text {pre }}=5, N_{\text {suf }}=32, N_{g}=37$. The channel model CM-2 with $\Omega_{0}=1, L_{h}=6$ and $K=6$ is adopted. $p(t)$ is a spectral raised cosine pulse with a filter span of $\left[-5 T_{s}, 5 T_{s}\right]$ and $t_{0}=5 T_{s}$ unless mentioned otherwise. We define SNR as the ratio of the channel input signal power to the receive filter output noise power.

Fig. 2 presents the average interference plus extra noise power $\Xi$ caused by the OLA method with several values of the OLA length $\left(M_{d}\right)$ and the timing offset. It shows that $\Xi$ increases significantly when the timing offset exceeds a certain range $[a, b]$, e.g., $a=-8, b=5$ for $M_{d}=20$, and this range increases with $M_{d}$ but $b$ is observed to be fixed around $t_{0} / T_{s}$. For a particular $M_{d}$, the timing offset should be within the corresponding range $[a, b]$ in order to minimize the interference plus extra noise power. This range is similar to the ISI-free timing offset range in CP-based OFDM systems but there are two differences. First, in CP-based systems the positive timing offsets definitely introduce interference, while in ZP OFDM systems the above best timing offset range includes some positive timing offsets. Second, for a timing offset within the corresponding best timing offset range, there is neither interference nor extra noise for CP-based systems but there is always extra noise and possibly interference for $\mathrm{ZP}$ systems. The plots in Fig. 2 also provide the information on how to set up the timing estimator parameters. For example, based on the plots in Fig. 2, our suggestion for the timing estimator proposed in [10] is to set the fixed timing adjustment parameter $\eta$ approximately equal to $\left[\sigma_{t}+\left(t_{0} / T_{s}\right)\right]$, where $\sigma_{t}$ is the standard deviation of the fine timing estimator, such that the timing offset is most of the time within the above range $[a, b]$. We set $\eta=8$ in our simulation with $t_{0} / T_{s}=5$.

In Fig. 3, we present the channel estimation MSE given in (21) versus the channel estimation window length $L^{\prime}$ for different values of the OLA length $M_{p}$ at SNR of $10 \mathrm{~dB}$. For our considered channel, the best parameter setting for the CIR estimation under perfect synchronization is $\left(\left[L^{\prime}\right]_{\text {perfect }}=\right.$ $\left.25,\left[M_{p}\right]_{\text {perfect }}=12\right)$. The MSE performance degradation due to the use of a smaller $L^{\prime}$ than $\left[L^{\prime}\right]_{\text {perfect }}$ is more significant than that due to the use of a larger $L^{\prime}$. When taking into account the random timing offset $\Delta_{k}$, the $L^{\prime}$ should be chosen larger than $\left[L^{\prime}\right]_{\text {perfect }}$ so that most of the time the CIR estimation window contains the first $\left[L^{\prime}\right]_{\text {perfect }}$ actual CIR taps. $M_{p}$ should also be increased accordingly. A good design is to set $L^{\prime}=\left[L^{\prime}\right]_{\text {perfect }}+\left\lceil\sigma_{t}-E\left[\Delta_{k}\right]\right\rceil$ and $M_{p}=\left[M_{p}\right]_{\text {perfect }}+\left\lceil\sigma_{t}-E\left[\Delta_{k}\right]\right\rceil$. With $t_{0} / T_{s}=5, N_{t}=132$, and $\eta=8$, we have $\left[\sigma_{t}-E\left[\Delta_{k}\right]\right\rceil \simeq 5$ and hence a good choice is $L^{\prime}=30$ and $M_{p}=18$. We observe that $\left[L^{\prime}\right]_{\text {perfect }}$ and $\left[M_{p}\right]_{\text {perfect }}$ are quite insensitive to the SNR. Hence we can use the same $L^{\prime}$ and $M_{p}$ for different SNRs in our design.

In Section V-B we showed that the interference plus extra noise power $\Xi$ varies with the OLA length $M_{d}$. Fig. 4(a) presents the plot of $\Xi / N$ versus $M_{d}$ at $\mathrm{SNR}=10 \mathrm{~dB}$ with different fixed timing offset values ${ }^{3}$ of $0,-5$ and -10 samples, respectively. We observe that with perfect timing the best $M_{d}$ is 13 , while with a timing offset $\Delta_{k}$ (a negative number due to the timing advancement) the best $M_{d}$ is $13-\Delta_{k}$. In Fig. 4(b) we present the corresponding uncoded BER simulation results for the QPSK modulated signals with one-tap frequencydomain equalization. The BER curves are consistent with the interference plus extra noise power curves we evaluated in Fig. 4(a). Note that choosing a larger value of $M_{d}$ is more robust to random timing offsets. Our suggestion for the choice of $M_{d}$ is the best $M_{d}$ value corresponding to a timing offset around $\left\lceil E\left[\Delta_{k}\right]-\sigma_{t}\right\rceil$. With $t_{0} / T_{s}=5, N_{t}=132$, and $\eta=8$, this timing offset would be around -5 and hence a good choice for $M_{d}$ is 18 . We observe that our designs give the same OLA length for $M_{p}$ and $M_{d}$.

Finally, in Fig. 5 we present the uncoded BER performance versus $M_{d}\left(=M_{p}\right)$ obtained with the synchronization method in [10] and the proposed channel estimation and equalization methods using $L^{\prime}=30$. This result shows the performance of the proposed channel estimation and equalization methods in the presence of random timing offsets and frequency offsets. We observe that the optimum values of $M_{d}$ slightly vary with different SNR values and our design of $M_{p}=M_{d}=18$ gives approximately the best result across the SNRs of interest. The BER curve for the time-frequency spread data is shown as "(spread)" in Fig. 5. We observe that at the cost of data rate reduction, the spreading improves the error performance significantly.

\section{CONCLUSIONS}

We have presented the channel estimation and one-tap frequency-domain equalization in ZP MB-OFDM systems. We have derived the covariance matrix of the sample-spaced channel impulse response vector by using the probability density functions of the UWB channel path delays, and using the covariance matrix we have presented how to optimize channel estimation and equalization methods in the presence of timing offset. The OLA window lengths of preamble and data can affect the system performance significantly. The best overlapadd window lengths for preamble and data are observed to be approximately the same and the use of the overlap-add length larger than the best value gives a more robust performance than the use of a smaller length in the presence of timing offset. Our optimization criteria also provide the information of the tolerable range of the timing estimation errors, which is valuable in timing estimator designs. The simulation results corroborate the effectiveness of our channel estimation and equalization methods and corresponding optimization criteria.

\footnotetext{
${ }^{3} \mathrm{We}$ only consider non-positive timing offsets since the timing adjustment yields negative timing offset most of the time.
} 


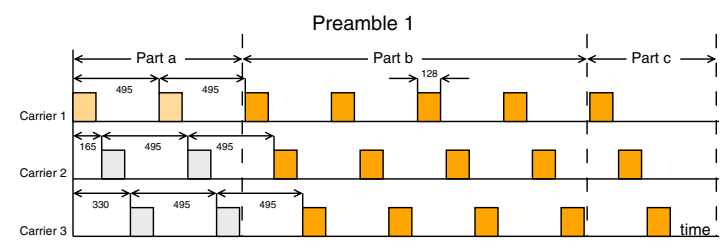

Fig. 1. The structure of the preamble pattern 1 from [1] and [2]

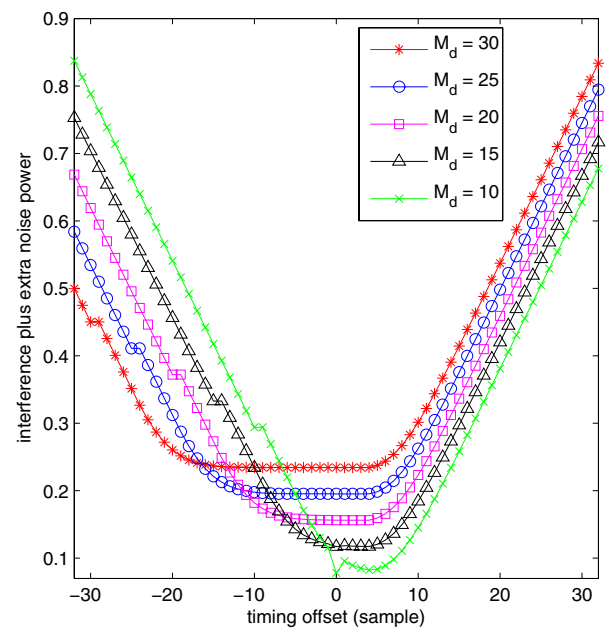

Fig. 2. The effects of timing offset and data OLA length $M_{d}$

\section{REFERENCES}

[1] Multi-band OFDM physical layer proposal for IEEE 802.15 Task Group 3a, IEEE Std., Mar. 2004.

[2] High rate ultra wideband PHY and MAC standard, ECMA Std., 2005.

[3] T. Pollet, M. Van Bladel, and M. Moeneclaey, "BER sensitivity of OFDM systems to carrier frequency offset and Wiener phase noise," IEEE Trans. Commun., pp. 191-193, Feb-Mar-Apr 1995.

[4] O. S. Shin, S. Ghazzemzadeh, L. Greenstein, and V. Tarokh, "Performance evaluation of MB-OFDM and DS-UWB systems for wireless personal area networks," in Proc. IEEE ICU, Sept. 2005, p. 6.

[5] O. Edfors, M. Sandell, J.-J. van de Beek, S. Wilson, and P. Borjesson, "OFDM channel estimation by singular value decomposition," IEEE Trans. Commun., vol. 46, no. 7, pp. 931-939, July 1998.

[6] Y. Li, J. Cimini, L.J., and N. Sollenberger, "Robust channel estimation for OFDM systems with rapid dispersive fading channels," IEEE Trans. Commun., vol. 46, no. 7, pp. 902-915, July 1998.

[7] Y. Li, A. Molisch, and J. Zhang, "Practical approaches to channel estimation and interference suppression for OFDM-based UWB communications," IEEE Trans. Wireless Commun., pp. 2317-2320, Sept. 2006.

[8] Y. Li, T. Jacobs, and H. Minn, "Frequency offset estimation for MBOFDM-based UWB systems," in Proc. IEEE ICC, vol. 10, June 2006, pp. 4729-4734.

[9] A. Molisch, J. Foerster, and M. Pendergrass, "Channel models for ultrawideband personal area networks," IEEE Wireless Communications, vol. 10, no. 6, pp. 14-21, Dec. 2003.

[10] T. Jacobs, Y. Li, H. Minn, and R. Rajatheva, "Synchronization in MBOFDM-based UWB systems," accepted in ICC 2007.

[11] H. Minn, V. Bhargava, and K. Letaief, "A robust timing and frequency synchronization for OFDM systems," IEEE Trans. Wireless Commun., vol. 2, no. 4, pp. 822-839, July 2003.

[12] - "A combined timing and frequency synchronization and channel estimation for OFDM," IEEE Trans. Commun., pp. 416-422, Mar. 2006.

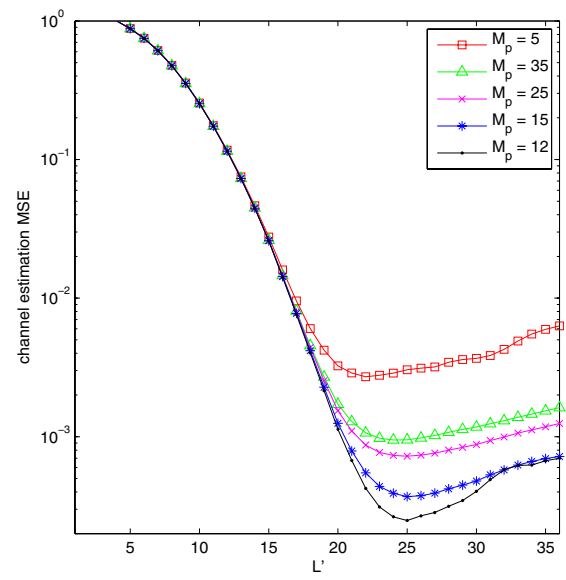

Fig. 3. The effects of CIR estimation length $L^{\prime}$ and preamble OLA length $M_{p}$ on the channel estimation MSE $(\mathrm{SNR}=10 \mathrm{~dB})$

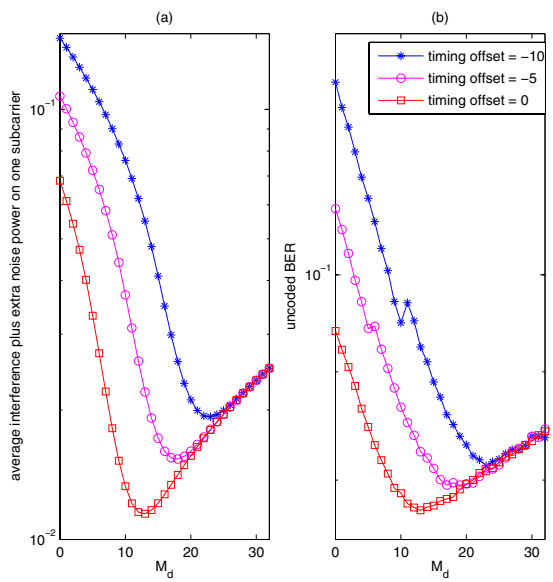

Fig. 4. The effect of data OLA length $M_{d}(\mathrm{SNR}=10 \mathrm{~dB})$. (a) Proposed design metric for $M_{d}$ (b) The uncoded BER performance versus $M_{d}$

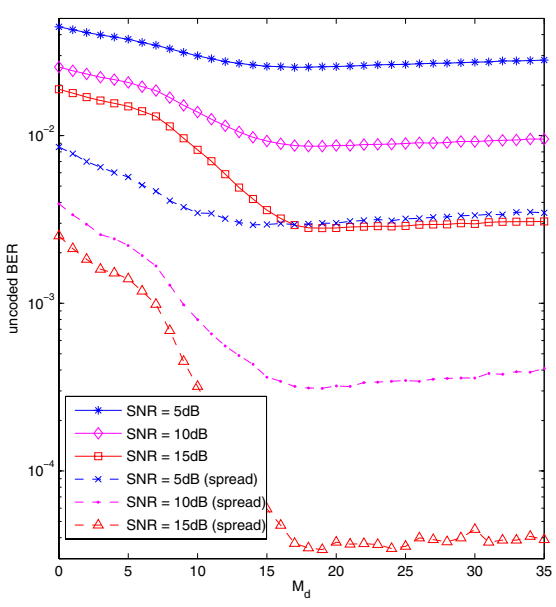

Fig. 5. The uncoded BER performance of the proposed methods with $L^{\prime}=$ 30 versus the OLA length $\left(M_{d}=M_{p}\right)$ (Synchronization method in [10] is used) 